\title{
BMJ Factors associated with depressive state OPen in patients with myasthenia gravis: a multicentre cross-sectional study
}

\author{
Yasushi Suzuki, ${ }^{1}$ Kimiaki Utsugisawa, ${ }^{2}$ Shigeaki Suzuki, ${ }^{3}$ Yuriko Nagane, ${ }^{2}$ \\ Masayuki Masuda, ${ }^{4}$ Chiaki Kabasawa, ${ }^{5}$ Yuko Shimizu, ${ }^{5}$ Hiroya Utsumi, ${ }^{4}$ \\ Shinichiro Uchiyama, ${ }^{5}$ Kazuo Fujihara, ${ }^{6}$ Norihiro Suzuki ${ }^{3}$
}

To cite: Suzuki Y, Utsugisawa K, Suzuki S, et al. Factors associated with depressive state in patients with myasthenia gravis: a multicentre cross-sectional study. BMJ Open 2011;1: e000313. doi:10.1136/ bmjopen-2011-000313

- Prepublication history for this paper is available online. To view these files please visit the journal online (http:// bmjopen.bmj.com).

Received 16 August 2011 Accepted 1 November 2011

This final article is available for use under the terms of the Creative Commons Attribution Non-Commercial 2.0 Licence; see http://bmjopen.bmj.com

\footnotetext{
${ }^{1}$ Department of Neurology, Sendai Medical Center, Sendai, Japan

${ }^{2}$ Department of Neurology, Hanamaki General Hospital, Hanamaki, Japan

${ }^{3}$ Department of Neurology, Keio University School of Medicine, Tokyo, Japan

${ }^{4}$ Department of Neurology, Tokyo Medical University, Tokyo, Japan

${ }^{5}$ Department of Neurology, Tokyo Women's Medical University, Tokyo, Japan ${ }^{6}$ Department of Neurology, Tohoku University Graduate School of Medicine, Sendai, Japan
}

Correspondence to Kimiaki Utsugisawa; kutsugi@s4.dion.ne.jp

\section{ABSTRACT}

Objectives: The objective of this study was to examine clinical factors associated with depressive state in patients with myasthenia gravis (MG).

Design: Cross-sectional study.

Setting and participants: We evaluated 287 consecutive cases of MG seen at six neurological centres located in Eastern Japan.

Outcome measures: All MG patients completed the Japanese version of the Beck Depression

Inventory-Second Edition (BDI-II). Disease severity was determined according to the MG Foundation of America (MGFA) quantitative MG score, MG activities of daily living scale and MG composite scale (MG composite). Clinical state following treatment was categorised according to MGFA postintervention status. Associations between detailed clinical parameters of MG and BDI-II score were then examined statistically.

Results: Mean BDI-II score for patients with MG $(11.0 \pm 8.1)$ did not differ substantially from and overlapped with that reported as the Japanese standard $(8.7 \pm 6.4)$. The mean +2 SDs for the Japanese standard is 21.5 , approximately equal to the cut-off level indicative of moderate or worse depression ( $>20$ points) in the original English version. We thus defined BDI-II $>21.5$ as depressive state, with a frequency of $13.6 \%$ in patients with MG. Multivariate logistic regression analysis revealed current dose of oral prednisolone (OR $1.09,95 \% \mathrm{Cl}$ 1.02 to $1.17 ; p=0.01$ ), unchanged MGFA postintervention status (OR $3.55,95 \% \mathrm{Cl} 1.18$ to $10.71 ; p=0.02)$, time since onset $(\mathrm{OR} 0.93,95 \% \mathrm{Cl}$ 0.87 to $0.99 ; p=0.03$ ) and MG composite (OR 1.16, $95 \% \mathrm{Cl} 1.00$ to $1.34 ; p=0.046$ ) as factors independently associated with depressive state in MG. Conclusions: Dose of oral corticosteroids appears to represent the major factor associated with depressive state in MG. Unchanged status despite treatment and early disease stage are also significant background factors for depressive state, along with disease severity.

\section{ARTICLE SUMMARY}

\section{Article focus}

- Background factors associated with depressive state in MG were statistically examined.

Key messages

- Dose of oral corticosteroids appears to represent the major factor associated with depressive state in MG. Unchanged status despite treatment and early disease stage are also significant background factors for depressive state, along with disease severity.

- Achieving early improvement of disease by adequate MG therapy without long-term use of higher dose oral corticosteroids may be important to avoiding depressive state in patients with MG.

Strengths and limitations of this study

- Strength: this study probably is the first to systematically and statistically examine the associations between detailed disease-related parameters of $M G$ and depressive state.

- Limitation: predictive modelling cannot be strictly performed on this cross-sectional sample. Weakness: the absence of social factors as variables.

\section{INTRODUCTION}

Individuals with myasthenia gravis (MG) face difficulty in maintaining daily activities and are required to cope with a chronic condition of weakness and fatigability of variable and fluctuating severity. ${ }^{12}$ Chronic and disabling disease conditions often lead to psychiatric consequences, such as anxiety and depressive disorders. ${ }^{12}$ The constant need for medications may decrease quality of life and cause psychological stress. ${ }^{1}{ }^{2}$ Depressive symptoms have long been suggested to be common in patients with MG and extent to which and how depressive symptoms are involved in MG has been a matter of some discussion. ${ }^{1-4}$ However, disease-specific psychopathological backgrounds remain undefined and results 
from psychological testing have been inconsistent. ${ }^{1-3}$ The frequency of depressive symptoms in patients with MG has also been inconsistently reported using different measures. ${ }^{1-3}$ Although an intricate relationship to MG appears to exist, previous studies were small scale and did not clearly show causal backgrounds of depressive symptoms in MG. ${ }^{12}$

The present study evaluated self-reported symptoms of depression in a sample of 287 patients with MG using a standardised measure, the Beck Depression InventorySecond Edition (BDI-II), ${ }^{5}{ }^{6}$ and systematically and statistically examined associations with detailed clinical parameters of MG. The purpose of this study was to clarify clinical factors associated with depressive state in patients with MG.

\section{PATIENTS AND METHODS}

Patients

This cross-sectional study was conducted at six neurological centres located in Eastern Japan (three in Tohoku district and three in Tokyo area, East Japan MG study group). We evaluated 287 consecutive non- demented patients with MG seen at Sendai Medical Center, Hanamaki General Hospital, Tohoku University Hospital, Keio University Hospital, Tokyo Medical University Hospital and Tokyo Women's Medical University Hospital from May until August 2010. To avoid a potential bias, we enrolled consecutive patients during short duration (3 months). Patients with any missing clinical data were excluded. All patients provided written informed consent and were subjected to analysis. Clinical background data for the 287 patients with MG are shown in table 1 .

The diagnosis of MG was based on clinical findings (fluctuating symptoms with easy fatigability and recovery after rest) with reductions in symptoms after intravenous administration of anticholinesterase, decremental muscle response to a train of low-frequency repetitive nerve stimuli or the presence of antibodies against the acetylcholine receptor of skeletal muscle (AChR-Ab) or against muscle-specific tyrosine kinase (MuSK-Ab). Single-fibre electromyography was not systematically examined. AChRAb-negative cases comprised 55 of 287 patients. Two of the 55 AChR-Ab-negative patients were MuSK-Ab-positive.

Table 1 Backgrounds of patients and correlations of clinical factors with BDI-II score

\begin{tabular}{|c|c|c|c|}
\hline Clinical factors & $\begin{array}{l}\text { Patient backgrounds } \\
\text { ( } 287 \text { in total) }\end{array}$ & Correlation $(95 \% \mathrm{Cl})$ & p Value \\
\hline Age (years) & $57.5 \pm 17.1$ & $-0.05(-0.16$ to 0.07$)$ & 0.200 \\
\hline Female* & $n=193$ & $0.19(0.07$ to 0.30$)$ & $0.001 \dagger$ \\
\hline Time since onset (years) & $8.9 \pm 8.3$ & $-0.08(-0.19$ to 0.04$)$ & 0.10 \\
\hline Age at onset (years) & $48.0 \pm 18.6$ & $-0.01(-0.13$ to 0.10$)$ & 0.41 \\
\hline Thymectomy* & $n=141$ & $0.02(-0.10$ to 0.13$)$ & 0.39 \\
\hline Thymoma* & $n=63$ & $0.06(-0.05$ to 0.18$)$ & 0.15 \\
\hline QMG & $7.0 \pm 5.1$ & $0.33(0.22$ to 0.43$)$ & $<0.0001 \dagger$ \\
\hline Ocular QMG & $1.7 \pm 1.8$ & 0.29 (0.17 to 0.39$)$ & $<0.0001 \dagger$ \\
\hline Bulbar QMG & $0.3 \pm 0.8$ & $0.14(0.02$ to 0.25$)$ & $0.01+$ \\
\hline MG composite & $5.8 \pm 5.7$ & 0.40 (0.30 to 0.50$)$ & $<0.0001 \dagger$ \\
\hline MG-ADL & $3.4 \pm 3.1$ & 0.39 (0.29 to 0.49$)$ & $<0.0001 \dagger$ \\
\hline MGFA classification (worst)* & $\begin{array}{l}\text { I/II/III/IV/V: } \\
68 / 125 / 60 / 12 / 22\end{array}$ & 0.17 (0.05 to 0.28$)$ & $0.002 \dagger$ \\
\hline Current dose of PSL (mg/day) & $4.6 \pm 5.9$ & $0.33(0.22$ to 0.43$)$ & $<0.0001 \dagger$ \\
\hline Maximum dose of PSL (mg/day) & $20.5 \pm 20.7$ & $0.02(-0.09$ to 0.14$)$ & 0.34 \\
\hline Calcineurin inhibitors ${ }^{*}$ & $\mathrm{n}=115$ & $0.07(-0.04$ to 0.19$)$ & 0.10 \\
\hline Crisis $^{*}$ & $\mathrm{n}=22$ & $0.02(-0.10$ to 0.14$)$ & 0.38 \\
\hline AChR-Ab positivity* & $\mathrm{n}=232$ & $-0.14(-0.25$ to -0.02$)$ & $0.01 \dagger$ \\
\hline Kv 1.4-Ab positivity* & $\mathrm{n}=37$ & $0.05(-0.08$ to 0.18$)$ & 0.22 \\
\hline Titin-Ab positivity* & $\mathrm{n}=53$ & $0.09(-0.07$ to 0.26$)$ & 0.10 \\
\hline MuSK-Ab positivity ${ }^{*}$ & $\begin{array}{l}n=2 \text { (of } 55 \text { AChR } \\
\text { Ab-negative patients) }\end{array}$ & Not determined & Not determined \\
\hline $\mathrm{CSR}^{*}$ & $\mathrm{n}=21$ & $-0.10(-0.22$ to 0.01$)$ & $0.04 \dagger$ \\
\hline$P^{*}$ & $\mathrm{n}=22$ & $-0.09(-0.20$ to 0.03$)$ & 0.08 \\
\hline $\mathrm{MM}^{*}$ & $\mathrm{n}=95$ & $-0.17(-0.28$ to -0.06$)$ & $0.002 \dagger$ \\
\hline $1^{*}$ & $n=91$ & $0.12(0.01$ to 0.23$)$ & $0.02 \dagger$ \\
\hline$U^{*}$ & $n=54$ & $0.13(0.02$ to 0.24$)$ & $0.01 \dagger$ \\
\hline$W^{*}$ & $\mathrm{n}=4$ & $0.03(-0.08$ to 0.15$)$ & 0.28 \\
\hline
\end{tabular}

*Pearson's correlation or Spearman's rank correlation.

+Variables entered into multivariate regression analysis (see the Results section in the text).

AChR-Ab, antibodies against acetylcholine receptor; BDI-II, Beck Depression Inventory-Second Edition; CSR, complete stable remission; I, improved; Kv1.4, voltage-gated potassium channel 1.4; MG, myasthenia gravis; MG-ADL, MG activities of daily living scale; MG composite, MG composite scale; MGFA, Myasthenia Gravis Foundation of America; MM, minimal manifestations; MuSK, muscle-specific tyrosine kinase; PR, pharmacologic remission; PSL, prednisolone; QMG, MGFA quantitative MG score; U, unchanged; W, worse. 


\section{Clinical parameters}

All patients completed the BDI-II, a 21-item self-administered questionnaire designed to detect symptoms of depression and assess the severity of depression. ${ }^{56}$ BDIII has been shown to represent a reliable and valid measure of depression. ${ }^{5}{ }^{6}$ According to standardised scoring guidelines in the original English version, scores were categorised as indicative of moderate (20-28 points) or severe depression ( $\geq 29$ points). ${ }^{5-7}$ The Japanese version of the BDI-II has also been validated, and the mean score of the Japanese standard population reported as $8.7 \pm 6.4,{ }^{8}$ somewhat lower than that of the original English version.

Clinical factors shown in table 1 were evaluated for each patient and entered into analysis. Clinical severity at study entry was determined by patients and participating neurologists according to the MG Foundation of America (MGFA) quantitative MG score (QMG) ${ }^{9}$ MG activities of daily living scale (MG-ADL) ${ }^{10}$ and MG composite scale (MG composite). ${ }^{11}$ Clinical state at study entry following treatment was categorised according to MGFA postintervention status. ${ }^{9}$ The worst condition for each patient was classified according to the MGFA classification. ${ }^{9}$ Prednisone and prednisolone (PSL) are the standard agents for oral corticosteroid therapy in MG, and PSL is generally used in Japan. Dose of oral PSL was evaluated for both the current and maximum dosage.

Serum AChR-Ab titres were estimated by radioimmunoassay using ${ }^{125} \mathrm{I}$ - $\alpha$-bungarotoxin, with levels $\geq 0.5 \mathrm{nM}$ regarded as positive. MG-specific autoantibodies to voltage-gated potassium channel (Kv1.4-Ab) were detected by immunoprecipitation assay using ${ }^{35}$ S-labelled cellular extracts as the antigen source, as described elsewhere. ${ }^{12}$ Autoantibodies to titin (titin-Ab) was detected using a commercially available ELISA (DLD Diagnostika, Hamburg, Germany). MuSK-Ab was measured using a commercially available radioimmunoprecipitation assay (RSR, Cardiff, UK).

\section{Statistics}

Correlations between clinical factors and BDI-II score were evaluated using Pearson's correlation for continuous variables or Spearman's rank correlation for categorical variables converted to numerical variables. Factors found to have a $p$ value of $<0.05$ in univariate analysis were entered into multivariate linear regression analysis.

Differences between the two groups were evaluated using the Mann-Whitney $\mathrm{U}$ test for continuous variables and the $\chi^{2}$ test for categorical variables. Clinical factors associated with depressive state indicated by BDI-II $>21.5$ were examined by logistic regression analysis. Clinical factors found to have a $\mathrm{p}$ value of $<0.05$ in univariate analysis were entered into multivariate logistic regression analysis to determine background factors independently associated with depressive state.

$p$ Values of $<0.05$ were considered statistically significant. All continuous data are expressed as mean \pm SD. Statistical analyses were performed using UNISTAT V.5.6 statistical software (Unistat, London, UK).

\section{RESULTS}

\section{Correlations of clinical factors to BDI-Il score}

Positive correlations to BDI-II score were found for female sex, total QMG, ocular QMG, bulbar QMG, MG composite, MG-ADL, MGFA classification at the worst condition, current dose of PSL, and Improved (I) and Unchanged (U) MGFA postintervention status (table 1). Negative correlations were seen for AChR-Ab-positivity, and Complete Stable Remission and Minimal Manifestations MGFA postintervention status (table 1). These clinical factors were entered into multivariate linear regression analysis, which revealed severity (MG composite, $\mathrm{p}=0.008$; MG-ADL, $\mathrm{p}=0.003$ ) and current dose of PSL $(p=0.0002)$ as factors with independent positive effects on BDI-II total score. However, as mean BDI-II score for the 287 patients with MG was 11.0 \pm 8.1 and did not differ substantially from and overlapped with that reported as the Japanese standard $(8.7 \pm 6.4),{ }^{8}$ determining background factors for depressive state in MG using a linear regression model was potentially inappropriate. We therefore conducted further examinations using another regression model.

\section{Factors associated with depressive state}

Mean +2 SDs for the Japanese standard BDI-II score is $21.5,{ }^{8}$ approximately equal to the cut-off level indicative of moderate or worse depression (BDI-II $>20$ ) in the original English version. ${ }^{5-7}$ We thus defined BDI-II $>21.5$ as indicating depressive state, with a frequency of $13.6 \%$ (39 of 287) among patients with MG in this study. In the present subjects, as no patients showed BDI$\mathrm{II}=21$, the total of 39 patients with depressive state using our definition (BDI-II >21.5) was identical to the result we would have achieved if a cut-off of BDI-II $>20$ had been used. Backgrounds of patients with or without depressive state and comparison between the two groups were shown in table 2.

Clinical factors associated with depressive state were examined by logistic regression analysis. Factors displaying a $\mathrm{p}$ value of $<0.05$ in univariate logistic regression analysis were time since onset, $\mathrm{QMG}$, ocular QMG, MG composite, MG-ADL, current dose of PSL, use of calcineurin inhibitors and $\mathrm{U}$ and Worse (W) MGFA postintervention status (table 3). These factors were entered into multivariate logistic regression analysis for determination of independent background factors associated with depressive state. Multivariate logistic regression analysis revealed current dose of PSL (OR $1.09,95 \%$ CI 1.02 to $1.17 ; \mathrm{p}=0.01)$, U of MGFA postintervention status (OR 3.55, 95\% CI 1.18 to 10.71; $\mathrm{p}=0.02$ ), time since onset (OR $0.93,95 \%$ CI 0.87 to 0.99 ; $\mathrm{p}=0.03$ ) and MG composite (OR 1.16, 95\% CI 1.00 to $1.34 ; \mathrm{p}=0.046)$ as independent background factors associated with depressive state in MG.

\section{DISCUSSION}

The present study examined associations between detailed parameters of MG and self-reported depressive 
Table 2 Comparison between patients with and without depressive state

\begin{tabular}{|c|c|c|}
\hline Clinical factors & $\begin{array}{l}\text { Patients with depressive } \\
\text { state }(n=39)\end{array}$ & $\begin{array}{l}\text { Patients without } \\
\text { depressive state }(n=248)\end{array}$ \\
\hline Age (years) & $52.3 \pm 18.0$ & $58.2 \pm 16.9$ \\
\hline Female & $\mathrm{n}=28$ & $\mathrm{n}=165$ \\
\hline Time since onset (years) & $6.1 \pm 6.2^{\star}$ & $9.3 \pm 8.4$ \\
\hline Age at onset (years) & $46.1 \pm 18.7$ & $48.3 \pm 18.6$ \\
\hline Thymectomy & $\mathrm{n}=12$ & $n=129$ \\
\hline Thymoma & $\mathrm{n}=7$ & $\mathrm{n}=56$ \\
\hline QMG & $9.9 \pm 6.6^{\star *}$ & $6.6 \pm 4.7$ \\
\hline Ocular QMG & $2.5 \pm 2.2^{*}$ & $1.6 \pm 1.7$ \\
\hline Bulbar QMG & $0.5 \pm 1.2$ & $0.3 \pm 0.7$ \\
\hline MG composite & $10.4 \pm 7.8^{* \star *}$ & $5.7 \pm 5.5$ \\
\hline MG-ADL & $5.3 \pm 4.0^{\star \star}$ & $3.1 \pm 2.9$ \\
\hline MGFA classification (worst) & I/II/III/IV/V: 7/13/14/2/4 & I/II/III/IV/V: 61/112/46/10/18 \\
\hline Current dose of PSL (mg/day) & $8.1 \pm 7.0^{\star \star \star}$ & $4.1 \pm 5.6$ \\
\hline Maximum dose of PSL (mg/day) & $20.4 \pm 21.6$ & $20.5 \pm 20.6$ \\
\hline Calcineurin inhibitors & $\mathrm{n}=19$ & $\mathrm{n}=96$ \\
\hline Crisis & $\mathrm{n}=4$ & $\mathrm{n}=18$ \\
\hline AChR-Ab positivity & $n=24$ & $\mathrm{n}=208$ \\
\hline Kv 1.4-Ab positivity & $\mathrm{n}=6$ & $\mathrm{n}=31$ \\
\hline Titin-Ab positivity & $\mathrm{n}=8$ & $n=45$ \\
\hline CSR & $\mathrm{n}=0$ & $n=21$ \\
\hline PR & $\mathrm{n}=1$ & $\mathrm{n}=21$ \\
\hline MM & $\mathrm{n}=4$ & $n=91$ \\
\hline I & $n=11$ & $\mathrm{n}=80$ \\
\hline U & $n=13$ & $n=41$ \\
\hline W & $n=2$ & $\mathrm{n}=2$ \\
\hline
\end{tabular}

symptoms and revealed current dose of oral PSL as the most significant background for depressive state in MG. In fact, long-term use of corticosteroids has been suggested to affect the brain and result in the development of depressive conditions. ${ }^{13-15}$ Complications of long-term corticosteroid use emerge at around $10 \mathrm{mg} /$ day of prednisone or PSL. ${ }^{13-15}$ Consistently, mean dose of oral PSL in patients with MG in depressive state was $8.1 \mathrm{mg} /$ day, significantly higher than that in patients without depressive state $(4.1 \mathrm{mg} /$ day; $\mathrm{p}<0.001 \mathrm{using}$ Mann-Whitney U test). Although oral corticosteroids represent the first-line and most common agents for immunosuppressive treatment of $\mathrm{MG}^{16}$ the present results indicate a possible need for attention to dose and associated depressive side effects. The constant need for medication itself might also cause psychological stress, but use of calcineurin inhibitors (ie, cyclosporine microemulsion and tacrolimus) was not associated with depressive state. In Japan, oral cyclosporine microemulsion (2.0-5.0 mg/ kg/day) was divided into two doses taken at intervals of $12 \mathrm{~h}$, and oral tacrolimus (3.0-4.0 mg/day) was given once a day. There may be some differences of prescription pattern among Japan and other countries.
BDI-II score for patients with MG did not differ substantially from and overlapped with that of the Japanese standard. The frequency of individuals with depressive state (BDI-II > 21.5) was $13.6 \%$ in this study, lower than that reported by Fisher et $a l^{7}$ They reported that moderate or worse depression (BDI-II >20 in the original English version) was observed in $33 \%(15 / 45)$ of patients with $\mathrm{MG}^{7}{ }^{7}$ The frequency of depressive symptoms in patients with MG has been inconsistent in previous reports. ${ }^{13}$ Anxiety or depressive disorders have been reported as more frequent among patients with MG than in the general population. ${ }^{4} 17$ Conversely, several authors have found no increased frequency of depression among patients with MG compared to the general population. ${ }^{18-20}$ Regarding such discrepancies, given that the present study showed depressive symptoms to be positively correlated with both severity of MG and corticosteroid dose, frequency of depressive patients with MG may alter depending on such background factors in subjects.

The present analysis revealed that unchanged status despite treatment and early disease stage are also significant background factors for depressive state, along with disease severity. These findings suggest that achieving 
Table 3 Clinical factors associated with depressive state (univariate logistic regression)

\begin{tabular}{|c|c|c|}
\hline Clinical factors & OR $(95 \% \mathrm{Cl})$ & p Value \\
\hline Age (years) & $0.98(0.96$ to 1.00$)$ & 0.060 \\
\hline Female & 1.41 (0.63 to 3.14$)$ & 0.41 \\
\hline $\begin{array}{l}\text { Time since } \\
\text { onset (years) }\end{array}$ & 0.94 (0.89 to 1.00$)$ & $0.041^{*}$ \\
\hline $\begin{array}{l}\text { Age at onset } \\
\text { (years) }\end{array}$ & 0.99 (0.97 to 1.01$)$ & 0.51 \\
\hline Thymectomy & $0.39(0.18$ to 1.24$)$ & 0.27 \\
\hline Thymoma & 0.91 (0.38 to 2.20$)$ & 0.84 \\
\hline QMG & 1.12 (1.05 to 1.19$)$ & $0.0007^{*}$ \\
\hline Ocular QMG & $1.26(1.05$ to 1.52$)$ & $<0.012^{*}$ \\
\hline Bulbar QMG & $1.36(0.94$ to 1.98$)$ & 0.10 \\
\hline MG composite & $1.14(1.08$ to 1.20$)$ & $<0.0001^{*}$ \\
\hline MG-ADL & 1.21 (1.09 to 1.35$)$ & $0.0003^{*}$ \\
\hline $\begin{array}{l}\text { MGFA } \\
\text { classification } \\
\text { (worst) }\end{array}$ & 1.19 (0.88 to 1.62$)$ & 0.26 \\
\hline $\begin{array}{l}\text { Current dose } \\
\text { of PSL (mg/day) }\end{array}$ & 1.09 (1.04 to 1.15$)$ & $0.0006^{*}$ \\
\hline $\begin{array}{l}\text { Maximum dose } \\
\text { of PSL (mg/day) }\end{array}$ & 1.00 (0.98 to 1.02$)$ & 0.97 \\
\hline $\begin{array}{l}\text { Calcineurin } \\
\text { inhibitors }\end{array}$ & 2.07 (1.01 to 4.27 ) & $0.048^{*}$ \\
\hline Crisis & $0.93(0.26$ to 3.29$)$ & 0.91 \\
\hline $\begin{array}{l}\text { AChR-Ab } \\
\text { positivity }\end{array}$ & $1.00(1.00$ to 1.01$)$ & 0.28 \\
\hline $\begin{array}{l}\text { Kv 1.4-Ab } \\
\text { positivity }\end{array}$ & $1.92(0.70$ to 5.26$)$ & 0.20 \\
\hline $\begin{array}{l}\text { Titin-Ab } \\
\text { positivity }\end{array}$ & $1.71(0.93$ to 3.14$)$ & 0.10 \\
\hline $\begin{array}{l}\text { CSR (no case } \\
\text { with BDI-II >21.5) }\end{array}$ & Not determined & $\begin{array}{l}\text { Not } \\
\text { determined }\end{array}$ \\
\hline PR & 0.30 (0.04 to 2.28$)$ & 0.24 \\
\hline MM & $0.54(0.24$ to 1.24$)$ & 0.15 \\
\hline I & 1.24 (0.59 to 2.60$)$ & 0.57 \\
\hline$U$ & 3.14 (1.46 to 6.77$)$ & $0.004^{*}$ \\
\hline W & 5.93 (1.27 to 27.73$)$ & $0.023^{*}$ \\
\hline
\end{tabular}

*Variables entered into multivariate logistic regression analysis (see the Results section in the text).

AChR-Ab, antibodies against acetylcholine receptor; CSR, complete stable remission; I, improved; Kv1.4, voltage-gated potassium channel 1.4; MG-ADL, MG activities of daily living scale; MG composite, MG composite scale; MGFA, Myasthenia Gravis Foundation of America; MM, minimal manifestations; MuSK, muscle-specific tyrosine kinase; PR, pharmacologic remission; PSL, prednisolone; QMG, MGFA quantitative MG score; $\mathrm{U}$, unchanged; W, worse.

early improvement of disease by adequate somatic therapy against MG may be important to avoid a depressive state. ${ }^{3}$ Psychiatric symptoms have also been noted to emerge temporarily in exacerbated patients with MG, reversing if adequate somatic therapy is given. ${ }^{3} 4$

Lack of formal evaluation of psychopathological characteristics by psychiatrists may represent a limitation to the present study. Patients with MG have been suggested to exhibit symptoms characteristically similar to the vegetative signs of affective disorders, which may interfere with correct assessment of mood if using only self-rating depression scales. ${ }^{19} 20$
In conclusion, dose of oral corticosteroids appears to represent the major factor associated with depressive state in patients with MG. Unchanged status despite treatment and early disease stage are also significant background factors for depressive state, along with disease severity. Achieving early improvement of disease by adequate MG therapy without long-term use of higher dose oral corticosteroids may be important to avoiding depressive state in patients with MG.

Funding This work was supported by a Neuroimmunological Disease Research Committee grant from the Japanese Ministry of Health, Labour and Welfare.

Competing interests None.

Patient consent Obtained.

Ethics approval All clinical information and blood samples were obtained after providing informed consent, and the study protocols were approved by the ethics committees of each institute.

Contributors Dr K Utsugisawa had full access to all of the data in the study, takes responsibility for the integrity of the data and the accuracy of the data analysis, and certifies all authors to meet the International Committee of Medical Journal Editors (ICMJE) criteria. Study concept and design was performed by YSu, KU, SS and YN. Acquisition of data was performed by YSu, $\mathrm{KU}, \mathrm{SS}, \mathrm{YN}, \mathrm{MM}, \mathrm{CK}, \mathrm{YSh}$ and HU. Analysis and interpretation of data was performed by YSu, KU, SS, SU, KF and NS. Drafting the article or revising it critically for important intellectual content was performed by YSu, KU, SS, YN, MM, CK, YSh, HU, SU, KF and NS. Final approval of the version to be published was performed by YSu, KU, SS, YN, MM, CK, YSh, HU, SU, KF and NS. Obtained funding and study supervision by KU, SS and YN.

Provenance and peer review Not commissioned; externally peer reviewed.

Data sharing statement No additional data available.

\section{REFERENCES}

1. Keesey JC. Does myasthenia gravis affect the brain? J Neurol Sci 1999;170:77-89.

2. Kulaksizoglu IB. Mood and anxiety disorders in patients with myasthenia gravis: aetiology, diagnosis and treatment. CNS Drugs 2007;21:473-81.

3. Köhler W. Psychosocial aspects in patients with myasthenia gravis. $J$ Neurol 2007;254(Suppl 2):Il/90-2.

4. Paradis CM, Friedman S, Lazar RM, et al. Anxiety disorders in a neuromuscular clinic. Am J Psychiatry 1993;150:1102-4.

5. Beck AT, Steer RA, Brown GK. Manual for Beck Depression Inventory-II. San Antonio, TX: Psychological Corporation, 1996.

6. Steer RA, Clark DA, Beck AT, et al. Common and specific dimensions of self-reported anxiety and depression: the BDI-II versus the BDI-IA. Behav Res Ther 1999;37:183-90.

7. Fisher J, Parkinson K, Kothari MJ. Self-reported depressive symptoms in myasthenia gravis. J Clin Neuromuscul Dis 2003;4:105-8.

8. Kojima M, Furukawa TA, Takahashi $\mathrm{H}$, et al. Cross-cultural validation of the Beck Depression Inventory-II in Japan. Psychiatry Res 2002;110:291-9.

9. Jaretzki A 3rd, Barohn RJ, Ernstoff RM, et al; Task Force of the Medical Scientific Advisory Board of the Myasthenia Gravis Foundation of America. Myasthenia gravis: recommendation for clinical research standards. Neurology 2000;55:16-23.

10. Wolfe GI, Herbelin L, Nations SP, et al. Myasthenia gravis activities of daily living profile. Neurology 1999;52:1487-9.

11. Burns TM, Conaway M, Sanders DB; MG Composite and MG-QOL15 Study Group. The MG Composite: a valid and reliable outcome measure for myasthenia gravis. Neurology 2010;74:1434-40.

12. Suzuki S, Utsugisawa $\mathrm{K}$, Nagane $\mathrm{Y}$, et al. Classification of myasthenia gravis based on autoantibody status. Arch Neurol 2007;64:1121-4.

13. Truhan AP, Ahmed AR. Corticosteroids: a review with emphasis on complications of prolonged systemic therapy. Ann Allergy 1989;62:375-91.

14. Keenan PA, Jacobson MW, Soleymani RM, et al. The effect on memory of chronic prednisone treatment in patients with systemic disease. Neurology 1996;47:1396-402. 
15. Brown ES, Suppes T. Mood symptoms during corticosteroid therapy: a review. Harv Rev Psychiatry 1998;5:239-46.

16. Meriggioli MN, Sanders DB. Autoimmune myasthenia gravis: emerging clinical and biological heterogeneity. Lancet Neurol 2009;8:475-90.

17. Magni G, Micaglio GF, Lalli R, et al. Psychiatric disturbances associated with myasthenia gravis. Acta Psychiatr Scand 1988;77:443-5.
18. Doering S, Henze T, Schüssler G. Coping with myasthenia gravis and implications for psychotherapy. Arch Neurol 1993;50:617-20.

19. Paul RH, Nash JM, Cohen RA, et al. Quality of life and well-being of patients with myasthenia gravis. Muscle Nerve 2001;24: 512-16.

20. Paul RH, Cohen RA, Goldstein JM, et al. Severity of mood, selfevaluative, and vegetative symptoms of depression in myasthenia gravis. J Neuropsychiatry Clin Neurosci 2000;12:499-501. 